\title{
Folding Dynamics of DNA G-Quadruplexes Probed by Millisecond Temperature Jump Circular Dichroism
}

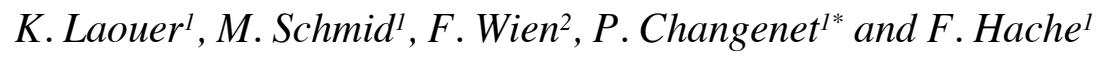

1- Laboratoire d'Optique et Biosciences, Ecole Polytechnique, CNRS-INSERM, Institut Polytechnique de Paris, 91128 Palaiseau Cedex, France.

2- L'Orme des Merisiers, Synchrotron SOLEIL, 91192 Gif sur Yvette, France

Corresponding author : pascale.changenet-barret@polytechnique.edu

\section{Supplementary information}

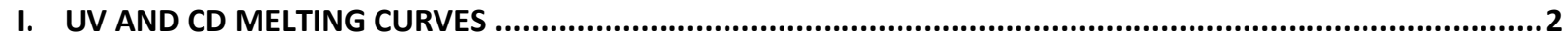

II. DETERMINATION OF THE MELTING TEMPERATURES...................................................................................

III. IN SITU MEASUREMENT OF TEMPERATURE ON THE T-JUMP SET-UP ........................................................4

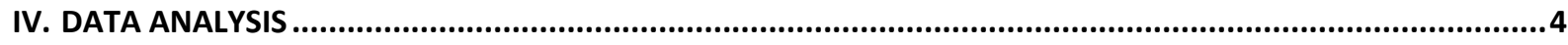

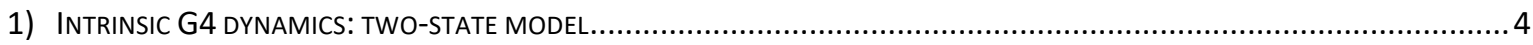

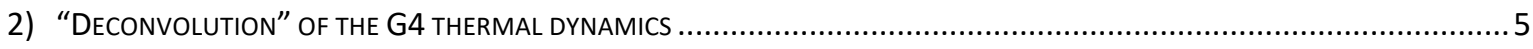

3) INSTANTANEOUS RISE AND DECAY IN THE ABSORPTION AND THE CD SIGNALS AFTER T-JUMP AND COOLING DOWN............... 6

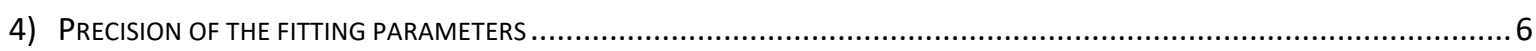

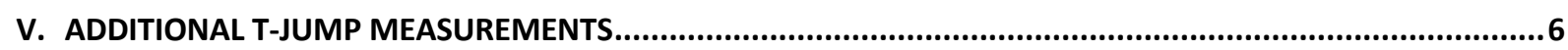

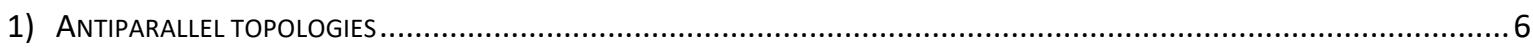

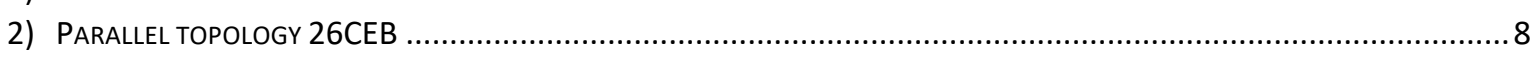

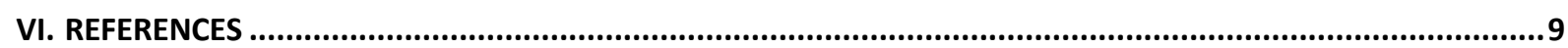




\section{UV and CD melting curves}

Figure S1 displays the UV melting curves registered by heating and cooling of the G4s, showing the absence of hysteresis in the denaturation and renaturation processes of DNA.
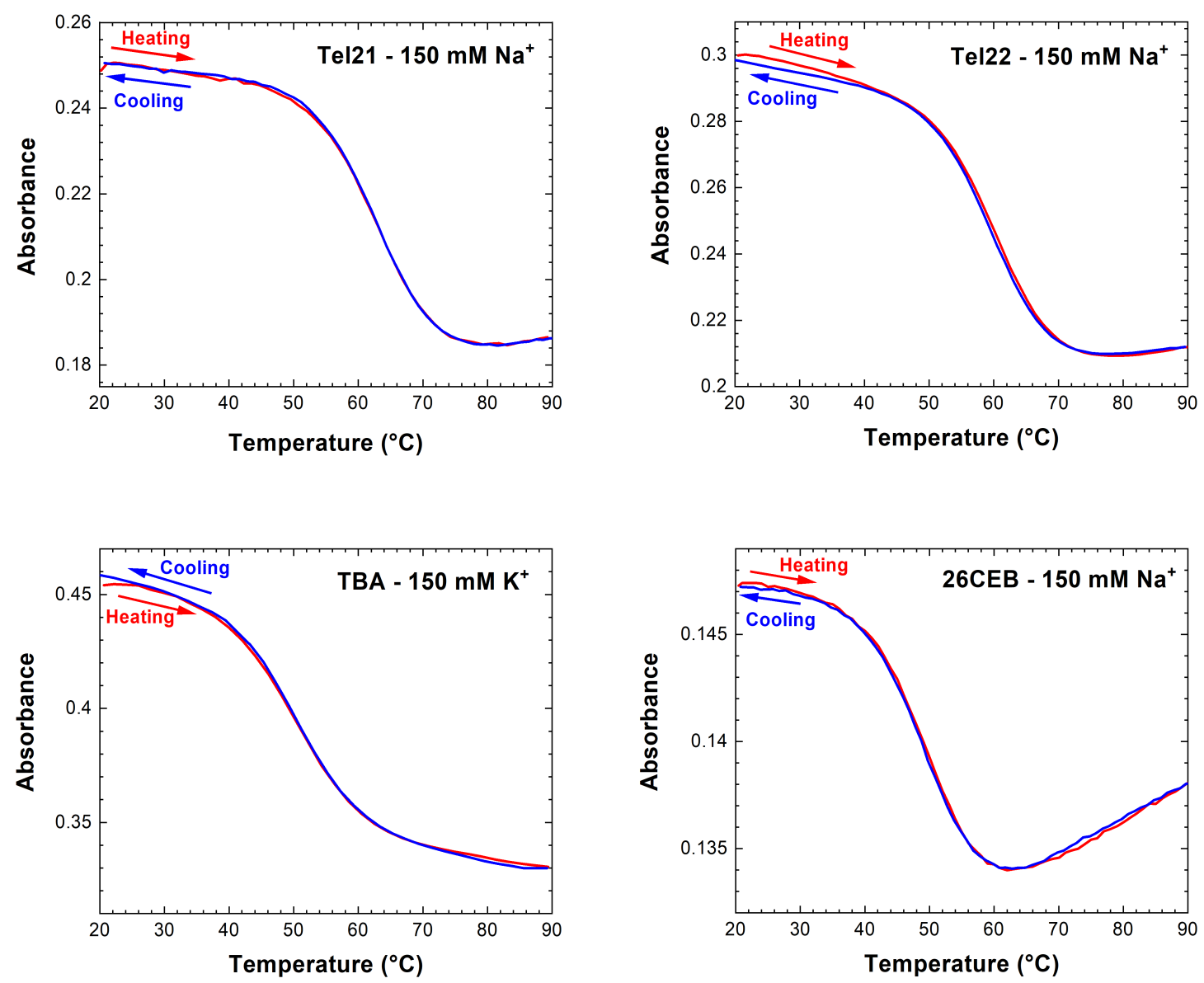

Figure S1: UV melting curves measured at $293 \mathrm{~nm}$ for solutions of Tel21, Tel22 and 26CEB with $150 \mathrm{mM} \mathrm{Na}^{+}$and a solution of TBA with $150 \mathrm{mM} \mathrm{K}^{+}$. Red and blue curves correspond to measurements for increasing and decreasing temperatures at a rate of $1^{\circ} \mathrm{C} / \mathrm{min}$, respectively.

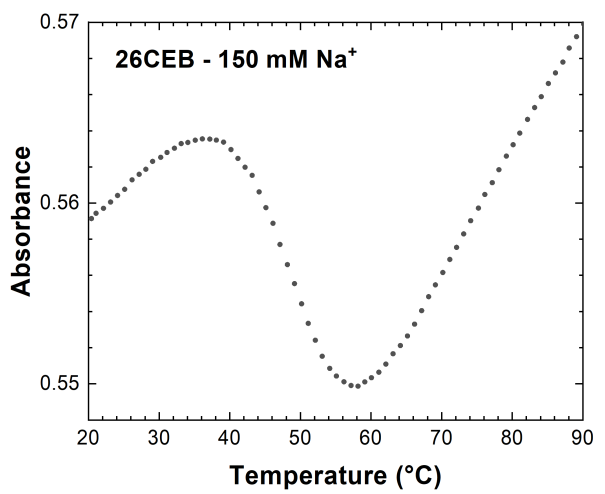

Figure S2: UV melting curve measured at a rate of $1{ }^{\circ} \mathrm{C} / \mathrm{min}$, at $265 \mathrm{~nm}$, for a solution of $26 \mathrm{CEB}$ with $150 \mathrm{mM} \mathrm{Na}^{+}$. 


\section{Determination of the melting temperatures}

The melting temperature $\left(T_{m}\right)$ has been determined at $293 \mathrm{~nm}$ or $265 \mathrm{~nm}$ from the UV and CD melting according the procedure described in ref. 1. In this regard, a simple two-state model assuming an equilibrium between one folded and unfolded state of DNA was used. For UV melting, the unfolded fraction of oligonucleotides can be calculated from the experimental data at $293 \mathrm{~nm}, O D(T)$, as follow:

$$
N_{u}(T)=L_{B}^{f}-O D(T) / L_{B}^{f}-L_{B}^{u}
$$

With $L_{B}^{u}$ and $L_{B}^{f}$, the baselines associated to the unfolded and folded oligonucleotides, respectively. The variation of the unfolded fraction of oligonucleotides as a function of temperature can be expressed as follows:

$$
N_{u}(T)=1 / 1+\exp \left[\frac{\Delta H^{0}}{R}\left(\frac{1}{T}-\frac{1}{T_{m}}\right)\right]
$$

with $\Delta H^{0}$ the enthalpy of the reaction and $R$, the gas constant. Such analysis is illustrated on Figure S3. Table S1 gathers the melting temperatures obtained from UV and CD melting.
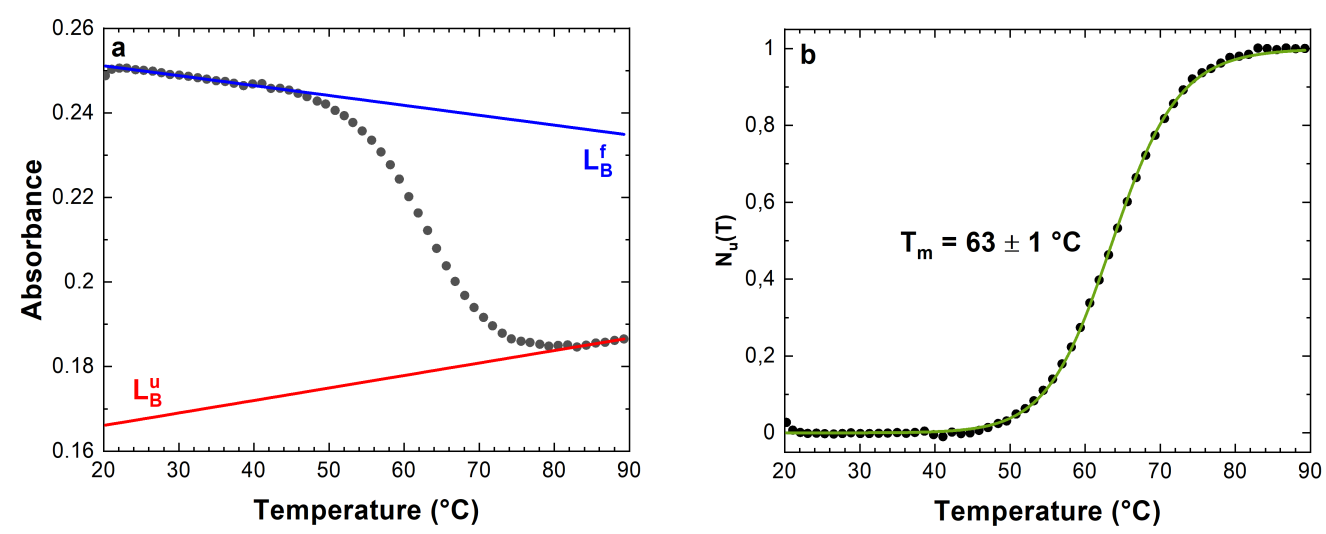

Figure S3: (a) UV melting curve measured at a rate of $1{ }^{\circ} \mathrm{C} / \mathrm{min}$, at $293 \mathrm{~nm}$, for a solution of Tel 21 with $150 \mathrm{mM} \mathrm{Na}^{+}$. Red and blue lines correspond to the baselines associated to unfolded and folded oligonucleotides, respectively. (b) Variation of the unfolded fraction of oligonucleotides, $N_{u}(T)$, as a function of temperature calculated from the UV melting curve. The green line illustrates the fit of $N_{u}(T)$ yielding a melting temperature of $63 \pm 1{ }^{\circ} \mathrm{C}$.

\begin{tabular}{|c|c|c|c|}
\hline Sequences & $\begin{array}{c}\mathbf{T}_{\mathbf{m}}\left({ }^{\circ} \mathbf{C}\right) \\
\mathbf{U V} \text { melting }\end{array}$ & $\begin{array}{c}\mathbf{T}_{\mathbf{m}}\left({ }^{\circ} \mathbf{C}\right) \\
\text { SRCD melting }\end{array}$ & $\begin{array}{c}\mathbf{T}_{\mathbf{m}}\left({ }^{\circ} \mathbf{C}\right) \\
\mathbf{C D} \text { melting } \\
\text { T-jump }\end{array}$ \\
\hline Tel21/150 mM Na ${ }^{+}$ & $63 \pm 1 @ 293 \mathrm{~nm}$ & $60 \pm 2 @ 293 \mathrm{~nm}$ & $61 \pm 1 @ 293 \mathrm{~nm}$ \\
\hline Tel21/80 $\mathbf{m M ~ N a}^{+}$ & $57 \pm 1 @ 293 \mathrm{~nm}$ & not measured & $57 \pm 1 @ 293 \mathrm{~nm}$ \\
\hline Tel22/150 $\mathbf{~ M M ~ N a}^{+}$ & $61 \pm 1 @ 293 \mathrm{~nm}$ & $58 \pm 2 @ 293 \mathrm{~nm}$ & $64 \pm 1 @ 293 \mathrm{~nm}$ \\
\hline TBA/150 $\mathbf{m M ~ K}^{+}$ & $51 \pm 1 @ 293 \mathrm{~nm}$ & $49 \pm 2 @ 293 \mathrm{~nm}$ & $52 \pm 1 @ 293 \mathrm{~nm}$ \\
\hline $\mathbf{2 6 C E B} / 150 \mathbf{m M ~ N a}^{+}$ & $50 \pm 1 @ 293 \mathrm{~nm}$ & $56 \pm 2 @ 265 \mathrm{~nm}$ & $54 \pm 1 @ 265 \mathrm{~nm}$ \\
\hline
\end{tabular}

Table S1: Melting temperatures determined from UV melting measurements with a double beam spectrophotometer, SRCD measurements at Soleil synchrotron and CD measurements on the T-jump set-up for Tel21, Tel22, TBA and 26CEB. 


\section{In situ measurement of temperature on the T-jump set-up}

The crucial point in T-jump experiments was to determine the temperature change of the sample when it is irradiated by the CW IR diode laser. In this regard, we carried out measurements of the absorbance changes of the phenol red $\mathrm{pH}$ indicator in Tris-HCl buffer $(100 \mathrm{mM}, \mathrm{pH}=7.4)$ with our double-beam spectrophotometer, as function of the temperature. The $\mathrm{pH}$ of the Tris buffer is known to be sensitive to the temperature. ${ }^{2}$ In the range between $15^{\circ} \mathrm{C}$ and $50^{\circ} \mathrm{C}$, the phenol red absorbance at $293 \mathrm{~nm}$ exhibits a linear decrease with a slope of $-0.0016 \pm 0.0002 /{ }^{\circ} \mathrm{C}$. With this value, we could determine the temperature change provoked by the IR irradiation on our T-jump set-up, with a precision of $1^{\circ} \mathrm{C}$, which corresponds to a change of 0.0003 in the phenol red absorbance.

We measured the kinetics of the temperature changes for initial temperatures going from 20 to $70{ }^{\circ} \mathrm{C}$ and for incident IR power varying between 0.6 to $1.7 \mathrm{~W}$ (i.e. corresponding to T-jump between 5 and $30^{\circ} \mathrm{C}$ ), at $293 \mathrm{~nm}$. All the kinetics were adjusted with a bi-exponential function with similar time constants. Typically, for increasing temperatures, in the time range between 1 to $5 \mathrm{~s}$, we obtained:

$$
\Delta T(t)=\Delta T_{\max }\left(1-A e^{-\Gamma_{1} t}-(1-A) e^{-\Gamma_{2} t}\right)
$$

with $1 / \Gamma_{1}=14 \mathrm{~ms}$ and $1 / \Gamma_{2}=1300 \mathrm{~ms}$ and $A=0.83$. For decreasing temperatures, in the time range between 5 and $10 \mathrm{~s}$, the fits yielded:

$$
\Delta T(t)=\Delta T_{\max }\left(B e^{-\Gamma{ }_{1} t}+(1-B) e^{-\Gamma{ }_{2} t}\right)
$$

with $1 / \Gamma_{1}{ }_{1}=30 \mathrm{~ms}$ and $1 / \Gamma_{2}{ }_{2}=1700 \mathrm{~ms}$ and $B=0.82 . \Delta T_{\max }$ is the maximal temperature change that depends on the incident IR power. Origin of the fast rising and decay component of the sample temperature have been discussed in details in ref. 3 and could be well reproduced by simulations accounting for the equilibration between the heat deposition and the thermal diffusion out of the sample. Note that the thermal conductivity of $\mathrm{CaF}_{2}$ being more than 16 times greater than that of water, the thermal diffusion out of the sample arises mainly from the sample cell windows and is actually barely sensitive to the solvent.

\section{Data analysis}

As shown previously, sample heating and cooling are biphasic processes that should be taken into account in order to extract the intrinsic dynamics of G4s from the experimental data.

\section{1) Intrinsic G4 dynamics: two-state model}

We consider first a simple two-state model considering the equilibrium between a folded and an unfolded state:

$$
N_{f} \stackrel{k_{u}}{\rightleftharpoons} N_{u}
$$


with $N_{f}$ and $N_{u}$ the population of folded and unfolded G4 DNA strands. Defining $\alpha_{f}$ and $\alpha_{u}$ the absorption coefficients of the folded and the unfolded states, the sample absorption reads:

$$
\alpha=N_{f}(t) \alpha_{f}+N_{u}(t) \alpha_{u}
$$

When the temperature changes, the fraction of unfolded population changes by $\Delta N_{u}(t)$, the change of the sample absorption as a function of time is expressed as follow:

$$
\Delta \alpha(t)=\Delta N_{u}(t) \cdot\left(\alpha_{u}-\alpha_{f}\right)
$$

with $\Delta N_{u}(t)=N_{u}(t)-N_{u}(0)$ corresponding to the temporal change of the unfolded fraction of population after the temperature jump. Introducing a unique relaxation rate $\gamma=k_{f}+k_{u}$, the dynamical equation governing the evolution of this change reads as:

$$
\begin{aligned}
\Delta N_{u}(t) / d t & =-\gamma\left[\Delta N_{u}(t)-\Delta N_{u}\left(T_{\text {final }}\right)\right] \\
\Delta N_{u}(t) & =\Delta N_{u}\left(T_{\text {final }}\right) \cdot\left(1-e^{-\gamma t}\right)(8)
\end{aligned}
$$

with $\Delta N_{u}\left(T_{\text {final }}\right)$, the difference in the fraction of the unfolded population before and after the T-jump, which can be readily obtained from the melting curves.

\section{2) "Deconvolution" of the G4 thermal dynamics}

In the above equations, the sample temperature instantaneously reaches its final value, which is not the case in the present T-jump experiments. To account for the biphasic rise of the sample temperature $(T(t))$ to its final value during the denaturation process, the following equation must be solved:

$$
d \Delta N_{u}(t) / d t=-\gamma\left[\Delta N_{u}(t)-\Delta N_{u}(T(t))\right]
$$

In order to fit our experimental data, $\Delta N_{u}(T(t))$ is replaced by the following biexponential phenomenological function derived from in situ measurements of the dynamics change of the sample temperature upon the T-jump (eq. 3). The analytical solution of eq. 9 can be therefore expressed as follow:

$$
\Delta N_{u}(t)=\Delta N_{u}\left(T_{\text {final }}\right)\left[1+\frac{p}{\gamma-\Gamma_{1}}\left(\Gamma_{1} e^{-\gamma t}+\gamma e^{-\Gamma_{1} t}\right)+\frac{1-p}{\gamma-\Gamma_{2}}\left(\Gamma_{2} e^{-\gamma t}+\gamma e^{-\Gamma_{2} t}\right)\right]
$$

Although eq. 10 looks quite complicated, it only depends on two fitting parameters, $\gamma$ and $\Delta N_{u}\left(T_{\text {final }}\right) . \mathrm{p}$ is a fixed fitting parameter corresponding to the fraction of unfolded species at the initial temperature, determined from the melting curves measured on the T-jump set-up. A similar treatment can be done for the renaturation process. Fits of the absorption and CD changes, after T-jump, were performed with the two following equations:

$$
\begin{gathered}
\Delta A(t)=\Delta a \times \Delta N_{u}(t) \\
\Delta C D(t)=C D(0)+\Delta c d \times \Delta N_{u}(t)
\end{gathered}
$$

with $\Delta a$ and $\Delta c d$, the amplitudes of the absorption and the CD changes after the T-jump. $C D(0)$ is the value of the CD signal before the T-jump for $\mathrm{t}<1 \mathrm{~s}$. 


\section{3) Instantaneous rise and decay in the absorption and the CD signals after $T$-jump and cooling down}

As it is clearly visible in Figure 6, prominent instantaneous rise and decay are observed in the absorption of 26CEB after T-jump and cooling down. Such effects in T-jump measurements are known to arise from non-cooperative events that also appear as the baselines in the UV melting curves of G4s. As shown in Figure S1, these non-cooperative effects are particularly strong for 26CEB at $265 \mathrm{~nm}$ but also visible in a lesser extend for the other sequences at $293 \mathrm{~nm}$. To take into account of these very fast changes in the absorption and the CD signals, after Tjump and cooling down, we added an additional term in the fits evolving proportionally to the sample temperature change.

\section{4) Precision of the fitting parameters}

For the thermal denaturation, all fits were performed with three parameters corresponding to the denaturation rate of $\mathrm{G} 4, \gamma$, the difference in the fraction of the unfolded population before and after the T-jump: $\Delta N_{u}\left(T_{\text {final }}\right)$ and the amplitude of the changes of the absorption and CD signals after the T-jump, $a$ and $c d$. A similar analysis was also carried out for the G4 thermal renaturation. The two amplitude parameters, $\Delta N_{u}\left(T_{\text {final }}\right), \Delta a$ or $\Delta c d$, are not completely independent and impair the precision of the fits. However, we estimate that the precision of the parameter $\gamma$ extracted from the fits is within $20 \%$. Furthermore, all fits being made in the same conditions, the relative values of $\gamma$ can be more precisely compared and discussed (see Figure 7 in the main text).

\section{Additional T-jump measurements}

\section{1) Antiparallel topologies}

Figure S4 compares the absorption and the CD changes averaged over a long acquisition time. The kinetics are found to be identical within our experimental accuracy.

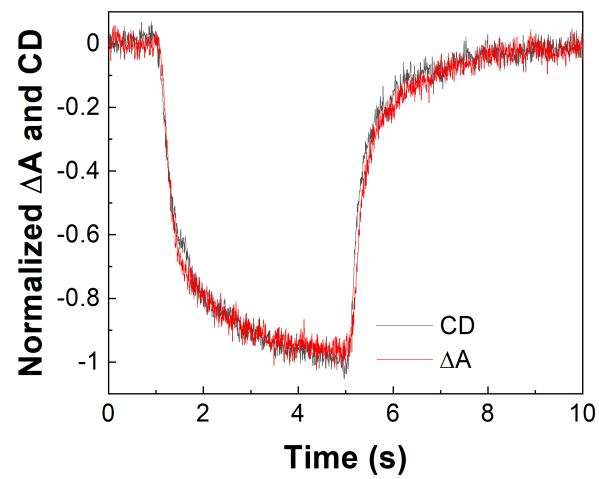

Figure S4: Comparison of the absorption and the CD changes measured for Tel21 with $80 \mathrm{mM} \mathrm{Na}$, following a T-jump of $10^{\circ} \mathrm{C}$ (initial temperature $=40^{\circ} \mathrm{C}$ ) after $1 \mathrm{~s}$ and a cooling down after $5 \mathrm{~s}$.

Figure S5 represents the raw experimental curves obtained for Tel21 (a) $80 \mathrm{mM}$ and (b) $150 \mathrm{mM} \mathrm{Na}^{+}$, together with the expected instantaneous response and the exponential fit (see main text). Lower and higher temperatures have also been measured with visible absorption 
changes, but most often the signals were too weak to yield meaningful adjustments. The extracted unfolding times are given in Table I in the main text.

(a)
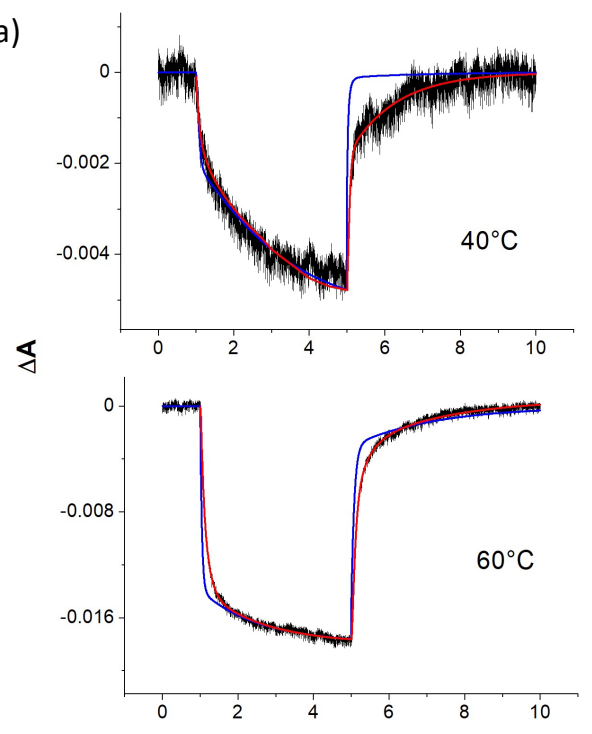
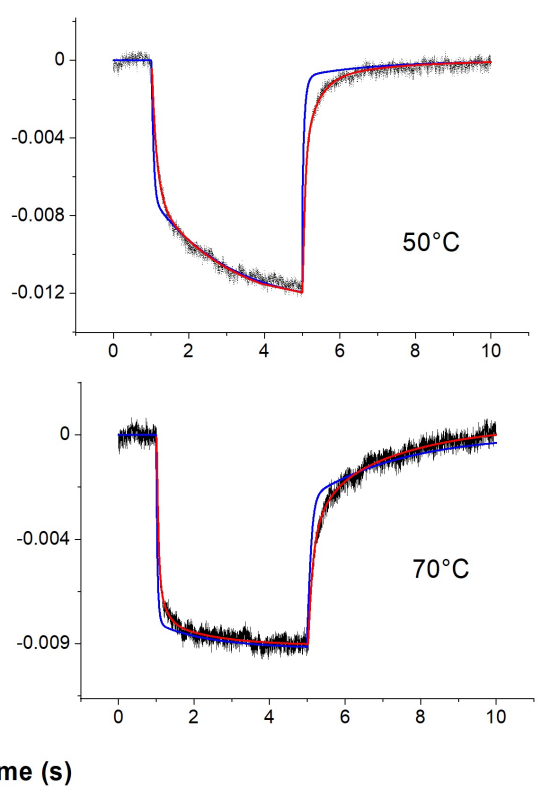

(b)
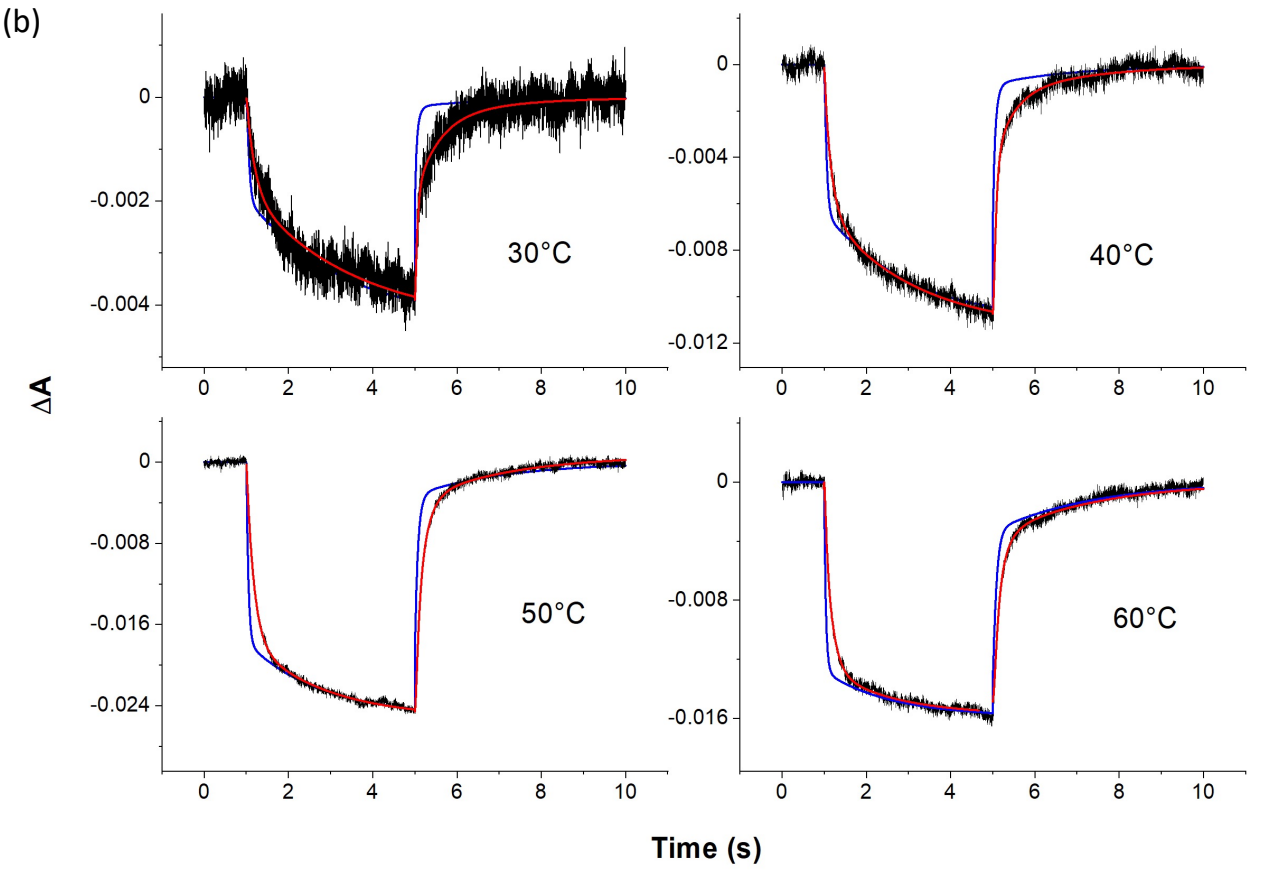

Figure S5: Absorption changes measured for Tel21 with (a) $150 \mathrm{mM}$ and (b) $80 \mathrm{mM} \mathrm{Na}^{+}$, following a T-jump of $10^{\circ} \mathrm{C}$ after $1 \mathrm{~s}$ and a cooling down after $5 \mathrm{~s}$, for various initial temperatures indicated in each panel. Blue lines correspond to the simulation of the solvent temperature change. Red lines correspond to the fits of the experimental data with a model of two interconverting species "convoluted" with the dynamics of the solvent temperature change. 


\section{2) Parallel topology 26CEB}

Figure S6 displays typical absorption changes measured at $266 \mathrm{~nm}$ for $26 \mathrm{CEB}$ with $150 \mathrm{mM}$ $\mathrm{Na}^{+}$, for an initial temperature of $40^{\circ} \mathrm{C}$, after a T-jump and subsequent cooling down of $10^{\circ} \mathrm{C}$. The most salient difference with the measurements of the antiparallel topologies performed at at $293 \mathrm{~nm}$ is the presence of the prominent instantaneous rise and decay, after T-jump at $1 \mathrm{~s}$ and cooling down at $5 \mathrm{~s}$, respectively. These fast changes that follow the temperature rise and decay of the sample are due to non-cooperative events that also appear in the equilibrium UV melting curves as the strong baselines at this specific wavelength in Figure S2. Denaturation and renaturation times extracted from the analytical fits of the absorption changes of $26 \mathrm{CEB}$, for different initial temperatures, are given in Table S2. The fits of the absorption and the CD changes in the time range of a few hundred milliseconds yielded comparable values providing evidence that the same cooperative processes are probed in both cases.

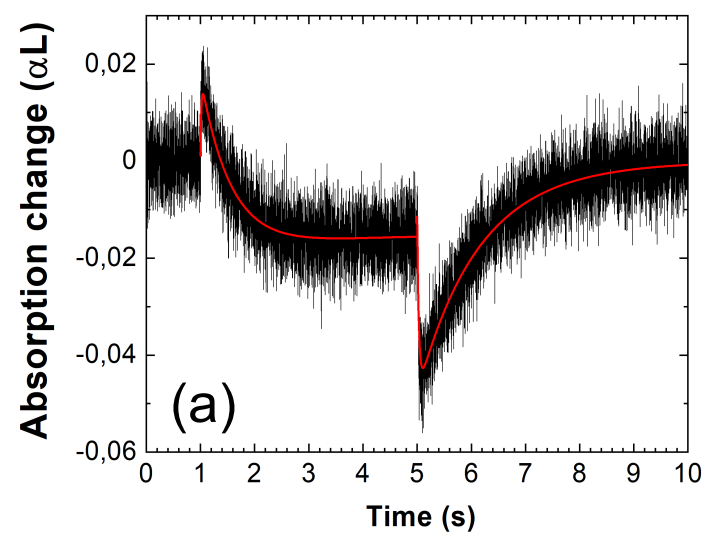

Figure S6: Absorption changes (black lines) at 260nm, measured for 26CEB in the presence of $150 \mathrm{mM}$ $\mathrm{Na}^{+}$at $40^{\circ} \mathrm{C}$, after a T-jump of $10^{\circ} \mathrm{C}$ after $1 \mathrm{~s}$ and subsequent cooling down to the initial temperature after $5 \mathrm{~s}$. Red lines correspond to the fits of the experimental data with a model of two interconverting species "convoluted" with the dynamics of the solvent temperature change.

\begin{tabular}{|c|c|c|c|c|}
\hline $\mathbf{T}\left({ }^{\circ} \mathbf{C}\right)$ & \multicolumn{2}{|c|}{$\boldsymbol{\tau}_{\text {Denat }}(\mathbf{m s})$} & \multicolumn{2}{c|}{$\boldsymbol{\tau}_{\text {Renat }}(\mathbf{m s})$} \\
\hline & Abs & $\mathrm{CD}$ & $\mathrm{Abs}$ & $\mathrm{CD}$ \\
\hline $\mathbf{2 0}$ & $n . m$. & 715 & 1586 & 1041 \\
\hline $\mathbf{3 0}$ & 682 & 554 & 1260 & 1085 \\
\hline $\mathbf{4 0}$ & 368 & 357 & 1039 & 936 \\
\hline $\mathbf{5 0}$ & 186 & 237 & 741 & 844 \\
\hline $\mathbf{6 0}$ & $n . m$. & 227 & 687 & 811 \\
\hline $\mathbf{7 0}$ & $n . m$. & 120 & $n . m$. & 646 \\
\hline
\end{tabular}

Table S2: Thermal denaturation and renaturation times ( $\tau_{\text {Denat }}$ and $\left.\tau_{\text {Renat }}\right)$ extracted from the fits of the absorption and the CD changes at $260 \mathrm{~nm}$, measured for 26CEB $\left(150 \mathrm{mM} \mathrm{Na}^{+}\right)$, after a T-jump and a cooling down of $10^{\circ} \mathrm{C}$, for different initial temperatures (n.m.: not measurable). 


\section{References}

1. Mergny, J. L.; Lacroix, L., Analysis of Thermal Melting Curves. Oligonucleotides 2003, 13, 515-537.

2. El-Harakany, A. A.; Abdel Halim, F. M.; Barakat, A. O., Dissociation Constants and Related Thermodynamic Quantities of the Protonated Acid Form of Tris-(hydroxymethyl)aminomethane in Mixtures of 2-Methoxyethanol and Water at Different Temperatures. Electroanal. Chem. 1984, 162, 285-305.

3. Ashwood, B.; Lewis, N. H. C.; Sanstead, P. J.; Tokmakoff, A., Temperature-Jump 2D IR Spectroscopy with Intensity-Modulated CW Optcal Heating. J. Phys. Chem. B 2020, 124 (39), 8665-8677. 